\title{
Patent Foramen Ovale: Story Closed?
}

\author{
Martin Köhrmann, ${ }^{\mathrm{a}, *}$ Peter D. Schellinger, ${ }^{\mathrm{b}, *}$ Georgios Tsivgoulis, ${ }^{\mathrm{c}, \mathrm{d}}$ Thorsten Steiner ${ }^{\mathrm{e}, \mathrm{f}}$ \\ aDepartment of Neurology, Essen University Hospital, Essen, Germany \\ 'Johannes Wesling Medical Center Minden, UK RUB, Minden, Germany \\ 'Second Department of Neurology, Attikon University Hospital, National and Kapodistrian University of Athens, Athens, Greece \\ ${ }^{d}$ Department of Neurology, University of Tennessee Health Sciences Center, Memphis, TN, USA \\ 'Department of Neurology, Frankfurt Hoechst Hospital, Frankfurt, Germany \\ fDepartment of Neurology, Heidelberg University Hospital, Heidelberg, Germany
}

The optimal treatment strategy for secondary prevention in patients with cryptogenic stroke and patent foramen ovale (PFO) has been a matter of controversy for decades. After three randomized trials failed to show a benefit of closure with an excess of complications in the interventional arm, two large recent trials suggest a benefit with regard of preventing further ischemic strokes. With this discrepancy in results it is important to discuss recent trials in detail and evolve an informed clinical approach for daily practice.

Keywords Stroke; Foramen ovale, patent; Intracranial embolism

\author{
Correspondence: Martin Köhrmann \\ Department of Neurology, Essen \\ University Hospital, University of \\ Duisburg-Essen, Hufelandstraße 55, \\ 45147 Essen, Germany \\ Tel: +49-201-723-6502 \\ Fax: +49-201-723-3962 \\ E-mail:Martin.koehrmann@uk-essen.de \\ Received: October 30, 2018 \\ Revised: November 27, 2018 \\ Accepted: December 11, 2018 \\ *These authors contributed equally to the \\ manuscript as first author.
}

\section{Introduction}

The prevalence of a patent foramen ovale (PFO) is estimated to be around $25 \%$ to $30 \%$ in the general population. ${ }^{1,2}$ Populationbased data regarding the prevalence in specific stroke populations are missing but observational data mainly derived from case control studies suggest an association between PFO (with or without atrial septal aneurysm [ASA]) and (cryptogenic) stroke in younger patient populations..$^{3-5}$ Recently, a population-based study from Oxfordshire (UK) indicated an association between right-to-left shunt and cryptogenic stroke in patients aged 60 years or older and highlighted that the population burden of PFO-associated events is substantial. ${ }^{6}$ Older trials and metaanalyses suggest higher stroke recurrence rates in younger stroke patients with PFO and ASA compared to patients without PFO or with isolated PFO (no ASA). ${ }^{7.8}$ However, overall recurrence rates are low and other meta-analyses failed to show such an association. ${ }^{9,10}$ Notably, patients with stroke and PFO do not have a higher annual risk of recurrent stroke in comparison with stroke patients without PFO (2.0\% vs. $2.4 \%)$ according to a meta-analysis evaluating the risk of PFO-associated stroke recurrence." The exact causal relationship, the role of PFO-morphology (e.g., small vs. large shunt volume) as well as the underlying stroke mechanism is still unclear. The role of the PFO-morphology and the fact that only a minority of affected patients have signs of deep vein thrombosis suggest that local processes may be more important than classical paradoxical embolism in the majority of patients. In addition, the low overall risk of recurrence in properly selected patient populations yield to conflicting results regarding risk factors for recurrent strokes. It is also often overlooked that besides the question of whether to close the PFO there are no firm data on the optimal medical treatment strategy. Finally, the definition of cryptogenic stroke with no defined diagnostic work-up and possible inclusion of patients with stroke mechanism very unlikely related to PFO is questionable. All of these factors lead to trials with heterogeneous patient populations and treatment arms making meta-analyses and pooled analyses more difficult and less valid (Table 1). 
Table 1. Overview of main inclusion criteria for PFO-trials

\begin{tabular}{|c|c|c|c|c|c|c|}
\hline & CLOSURE I 2012 & PC-trial 2013 & RESPECT 2013 & REDUCE 2017 & CLOSE 2017 & DEFENSE 2018 \\
\hline Number & $\begin{array}{l}909 \text { (closure 447, } \\
\text { medical 462) }\end{array}$ & $\begin{array}{l}414 \text { (closure 204, } \\
\text { medical 210) }\end{array}$ & $\begin{array}{l}980 \text { (closure } 499, \\
\text { medical } 481)\end{array}$ & $\begin{array}{l}664 \text { (closure } 441 \\
\text { medical 223) }\end{array}$ & $\begin{array}{l}663 \text { (closure 238, AP } \\
238, \text { OAC 187) }\end{array}$ & $\begin{array}{l}120 \text { (closure 60, medical } \\
60 \text { ) }\end{array}$ \\
\hline \multicolumn{7}{|l|}{ Inclusion criteria } \\
\hline Age (yr) & $18-60$ & $<60$ & $18-60$ & $18-60$ & $18-60$ & $18-80$ \\
\hline Disease & Stroke/TIA $<6$ mo & Stroke/TIA & Stroke/TIA $<7$ mo & Stroke $\leq 3 \mathrm{mo}$ & Stroke $\leq 6 \mathrm{mo}$ & Stroke $\leq 3 \mathrm{mo}$ \\
\hline $\begin{array}{l}\text { Other causes than } \\
\text { PFO ruled out by } \\
\text { appropriate inves- } \\
\text { tigations }\end{array}$ & To be excluded & $\begin{array}{l}\text { To be clinically and } \\
\text { radiologically ex- } \\
\text { cluded }\end{array}$ & To be excluded & To be excluded & $\begin{array}{l}\text { Other causes than PFO } \\
\text { ruled out by appropri- } \\
\text { ate investigations }\end{array}$ & $\begin{array}{l}\text { Other causes than PFO } \\
\text { ruled out by appropri- } \\
\text { ate investigations }\end{array}$ \\
\hline PFO & & & & & PFO with $A S A>10 \mathrm{~mm}$ & $\begin{array}{l}\text { High-risk PFO } \geq 2 \mathrm{~mm} \\
\text { and/or ASA } \geq 10 \mathrm{~mm}\end{array}$ \\
\hline Right-to-left shunt & $\begin{array}{l}\text { TEE and Valsalva } \\
\text { bubble study }\end{array}$ & $\begin{array}{l}\text { TEE or color Dop- } \\
\text { pler flow imaging }\end{array}$ & $\begin{array}{l}\text { TEE or color Doppler } \\
\text { flow imaging }\end{array}$ & $\begin{array}{l}\text { At rest or during Val- } \\
\text { salva (TEE, bubble) }\end{array}$ & $\begin{array}{l}>30 \text { microbubbles (TTE, } \\
\text { TEE) }\end{array}$ & TEE \\
\hline
\end{tabular}

PFO, patent foramen ovale; PC, Percutaneous Closure of PAtent Foramen Ovale in Cryptogenic Embolism; RESPECT, Randomized Evaluation of Recurrent Stroke Comparing PFO Closure to Established Current Standard of Care Treatment; AP, antiplatelet; OAC, oral anticoagulation; TIA, transient ischemic attack; TEE, transesophageal echocardiography; ASA, atrial septal aneurysm; TE, transthoracic echocardiography.

\section{Earlier trials: CLOSURE, PC-trial, RESPECT}

\section{CLOSURE I}

Evaluation of the STARFlex Septal Closure System in Patients with a Stroke and/or Transient Ischemic Attack due to Presumed Paradoxical Embolism through a Patent Foramen Ovale (CLOSURE I) randomized 909 patients between 18 to 60 years with PFO and cryptogenic stroke or transient ischemic attack (TIA) within the last 6 months to receive either medical therapy or PFO-closure using the STARFlex-system (NMT Medical, Boston, MA, USA). ${ }^{12}$ Best medical treatment was at the discretion of the investigator and consisted of aspirin (325 mg daily) or anticoagulation with warfarin (international normalized ratio [INR] 2-3). In the interventional arm patients received dual platelet therapy (81 or $325 \mathrm{mg}$ aspirin and $75 \mathrm{mg}$ clopidogrel) for 6 months followed by an aspirin monotherapy. Patients were followed for 2 years. Recurrence rates were comparable in both arms (2.9\% with PFO-closure vs. $3.1 \%$ in the medical arm). Closure was technically successful in $86 \%$ but procedure related complications were significant (3.2\%), and four patients had thrombi at the device detected at 6 months follow-up echocardiography. In addition, $5.7 \%$ of patients had new onset of atrial fibrillation (compared to $0.7 \%$ in the medical arm). Major bleeding events were numerically but non-significantly more common in the interventional arm (2.6\% vs. $1.1 \%, P=0.11$ ). Presence of an ASA as well as initial shunt size were not associated with higher risk of recurrence in this trial. After the results of the trial the device was withdrawn and the company went for bankruptcy.

\section{PC-trial}

The Clinical Trial Comparing Percutaneous Closure of Patent Foramen Ovale (PFO) Using the Amplatzer PFO Occluder with Medical Treatment in Patients with Cryptogenic Embolism (PC Trial) was initiated out of the Inselspital in Bern, Switzerland, and conducted in 29 sites in Europe, Canada, Brazil, and Australia. ${ }^{13}$ Overall 414 patients with cryptogenic stroke, TIA or systemic embolism and PFO below 60 years were randomized to receive closure of the PFO using the Amplatzer PFO Occluder (St. Jude Medical, St. Paul, MN, USA) or medical therapy (platelet inhibitors and/or oral anticoagulation [OAC]). The medical therapy was at the discretion of the treating physician. The primary endpoint was a composite of death, nonfatal stroke, TIA, or peripheral embolism. All endpoints were assessed blinded to the treatment (prospective randomized open blinded end-point [PROBE]-design). After a mean duration of follow-up of 4.1 years there was no difference in the primary endpoint (7/204 device group [3.4\%] vs. 11/201 medical group [5.2\%]; hazard ratio $[\mathrm{HR}], 0.63 ; 95 \%$ confidence interval $[\mathrm{Cl}], 0.24$ to 1.62 ; $P=0.34)$. None of the secondary endpoints were in favor of PFO-closure and the authors concluded that there was no benefit of PFO-closure compared to medical management alone.

\section{RESPECT-PFO-trial}

The Randomized Evaluation of Recurrent Stroke Comparing PFO Closure to Established Current Standard of Care Treatment (RESPECT)-trial was performed in 69 sites in the USA and Canada. ${ }^{14}$ In total 980 patients with a mean age of 45.9 years were randomized to receive PFO-closure again with the Amplatzer PFO Occluder or medical therapy alone. Antithrombotic therapy was 
at the discretion of the treating physician and mainly consisted of antiplatelet therapy ( $75 \%$ vs. $25 \%$ OAC). The trial was event driven and the main analysis was planned and conducted after 25 adjudicated endpoints were observed. A priori superiority of closure was set if at least 19 of these 25 endpoints occurred in the medical arm. Due to a very high dropout rate mainly in the medical arm overall treatment exposure of both arms were significantly different $(1,375$ patient-years in the closure-group vs. 1,184 patient-years in the medical group, $P=0.009$ ). In the medical arm 83/481 (17.3\%; 50/83 withdrew consent, 27 lost to follow-up) patients discontinued the trial, compared to $48 / 499$ (9.6\%; 18 withdrew consent, 19 lost to follow-up) in the closure-group. In addition, 37 patients (7.4\%) in the closure arm received no device leaving 462 treated patients. In the intention-to-treat (ITT) analysis 16 endpoint events occurred in the medical arm and nine in the closure-group. All observed endpoint were nonfatal ischemic strokes. However, due to the imbalance of treatment exposure of the two groups the raw-count analysis was deemed invalid. The time-to-event analysis using the ITT population demonstrated a $\mathrm{HR} 0.49(95 \% \mathrm{Cl}, 0.22$ to 1.11; $P=0.08$ ), thus failing to reach superiority. Overall, serious adverse events were also not different between the groups, procedure- or device-related serious events occurred in 21/499 patients (4.2\%). Several analyses using pre-specified per-protocol and modified as-treated cohorts were performed to demonstrate a treatment benefit for closure. These analyses will be discussed later in the article.

\section{RESPECT-long-term}

In 2017, long-term follow-up data of the RESPECT-PFO-trial were published after a median follow-up of 5.9 years. ${ }^{15}$ Again, treatment exposure between the two groups was unequal $(3,141$ patient-years in the closure group vs. 2,669 patient years in the medical group). Overall recurrence rates were low. During the follow-up 0.58 events per 100 patient-years were observed in the device group compared to 1.07 events per 100 patient-years in the medical group, yielding a HR of 0.55 with a wide $95 \% \mathrm{Cl}(0.31$ to 0.99$)$ and a borderline statistical significance with a $P$-value of 0.046 . It has to be noted though that as per regulatory requests or regulations, two previous follow-up analyses had been carried out after the first, primary publication of the results (see supplement of the reference 15). Both had not shown a difference of both arms. Regarding safety, pulmonary embolism occurred with a rate of 0.41 per 100 patient-years in the device group and 0.11 per 100 patient-years in the medical arm ( $\mathrm{HR}, 3.48 ; 95 \% \mathrm{Cl}, 0.98$ to $12.34 ; P=0.04)$, thus almost precisely balancing the benefit in the primary endpoint. Unfortunately, this fact is not mentioned in the abstract (probably due to word count restrictions) and is only highlighted in the discussion of the manuscript.

\section{Coping with these "negative results"}

\section{Denial}

The first German news released by St. Jude Medical after presentation of the initial RESPECT-trial results stated that "the RESPECT-trial proofs the clinical risk-optimization in the prevention of cryptogenic stroke" and that "the results clearly speak in favor of closure using the Amplatzer PFO Occluder instead of just medical therapy" (reference available with the authors). But advertisement is not restricted to industry in modern medicine. On the homepage of the Department of Cardiology of the Inselspital (Bern, Switzerland), the PC-trial (remember $P$-value for primary endpoint 0.37 and for ischemic stroke 0.79 ) was interpreted "closure was more than equal compared to medical therapy and within the trial 4 out of 5 strokes could have been prevented with closure." Furthermore, "closure can be performed in an outpatient setting, does not need anesthesia, is basically risk-free and the patient is able to do sports immediately after the intervention" (reference with the authors).

\section{Recalculating}

Several secondary analyses were undertaken using the RESPECT-trial data-some of them pre-specified. ${ }^{14}$ Starting with the initially planned IT-raw-count analysis, that was deemed invalid due to the high drop-out rate mainly in the medical arm, per protocol and as treated Kaplan-Meier analyses were calculated. Compared to the ITT population 25 patients were removed from the interventional arm to form the as-treated population for several reasons. Out of these four (16\%) had an outcome event, thus by far exceeding the expected background stroke risk in the medical population, clear signs of a bias introduced in the analysis (see supplement of the reference 14). Furthermore, eight patients moved from the interventional arm into the medical arm in the as-treated population. One of them (12.5\%, again a high rate) had an outcome event that was therefore subtracted from the interventional arm and added to the medical arm. Overall, the subtraction of these population with obviously particularly high risk seems troubling. As discussed above, in total four-time points of follow-up were performed for the RESPECT-trial, with the last one being the only one yielding positive results (though with an upper 95\% $\mathrm{Cl}$ of 0.999).

\section{Last exit: meta-analysis}

It is very common today to undertake meta-analyses as well as 
"pooled analyses" after addition of each new study regarding a specific topic regardless of differences of population, design or definitions. ${ }^{16}$ Several meta-analyses were undertaken using the data of these three "negative" trials and some of them came up with a positive overall outcome. In a well performed pooled analysis of the trials adjusted analysis yielded borderline results, but limiting the analysis to just the studies using the disc occluder device only (RESPECT, PC-trial) results were positive after also changing the endpoint from the initial composite to an ischemic stroke only endpoint. ${ }^{16}$

\section{The initial FDA-process}

On May 24th, 2016 the U.S. Food and Drug Administration (FDA) Circulatory System Device Panel met to advice for the clearance of the Amplatzer PFO Occluder System. Concerns were expressed regarding the higher rates of deep vein thrombosis and pulmonary embolism as well as signs for higher rates of arrhythmias in the interventional groups. Also, high dropout and cross-over rates were acknowledged. The panel recommended that these safety concerns should be monitored in post-approval studies and that the label should include recommendations for careful pre-interventional diagnostics and selection of cryptogenic stroke patients. Finally, the panel voted 15 versus 1 that the data show reasonable assurance for safe use, 9 versus 7 that the occluder is effective in this indication, and 11 versus 5 that benefits outweigh the risks. Not surprisingly interventional cardiologists on the panel were almost unanimously in favor of recommending approval, while neurologists all voted "no" on efficacy. Interestingly, both statisticians on the panel voted "no" on efficacy and risk/benefit.

On October 28th, 2016 the device was approved by the FDA.

\section{New trials}

During the European Stroke Organisation Conference 2017 in Prague two long awaited new PFO trials were presented and simultaneously published. ${ }^{17,18} \mathrm{~A}$ third trial was also presented and published in 2018. ${ }^{19}$

\section{GORE-REDUCE}

The REDUCE trial was a company-sponsored (WL Gore \& Associates, Inc., Newark, DE, USA) study that enrolled 664 patients (age 18 to 59 years; mean age 45.2 years) from December 2008 to February 2015 with cryptogenic stroke and PFO in a 2:1 manner to receive closure of the PFO using either the HELEX Septal Occluder (WL Gore \& Associates, Inc.) or Cardioform Septal Occluder (WL Gore \& Associates, Inc.) or antiplatelet therapy alone..$^{18}$ The mean follow-up was 3.2 years. By design, the majority of patients had moderate or large interarterial shunts, defined as 6 to 25 microbubbles (moderate) and more than 25 microbubbles (large). Initial lone primary end-point was freedom from clinical evidence of ischemic stroke through at least 24 months. After enrollment of 467 patients an additional co-primary endpoint (previously a secondary endpoint) was introduced as a composite of clinical stroke or silent brain infarction detected on 24-month magnetic resonance imaging (MRI). In the ITT population event rates were 0.39 strokes per 100 patient-years in the closure group and 1.71 strokes per 100 patient-years in the antiplatelet arm $(\mathrm{HR}, 0.23 ; 95 \% \mathrm{Cl}, 0.09$ to 0.62; $P=0.002$ ). The co-primary endpoint for silent MRI-infarctions was also positive (relative risk, $0.51 ; 95 \% \mathrm{Cl}, 0.29$ to 0.91 ; $P=0.04)$. Regarding safety, $3.9 \%$ of patient in the interventional arm had serious device (device-dislocation, device-related thrombosis, aortic dissection) or procedure related adverse events. While there was no difference in serious bleeding events or deep vein thrombosis/pulmonary embolism, 6.6\% of patients in the closure-arm compared to only $0.4 \%$ in the medical arm developed peri-procedural atrial fibrillation or flutter $(P<0.001)$.

\section{CLOSE-trial}

The Patent Foramen Ovale Closure or Anticoagulants versus Antiplatelet Therapy to Prevent Stroke Recurrence (CLOSE) study was an investigator initiated open label trial randomizing patients 16 to 60 years in a 1:1:1 ratio to receive PFO-closure plus long-term antiplatelet therapy (PFO-closure group), antiplatelet therapy alone (antiplatelet group), or OAC (anticoagulation group). ${ }^{17}$ Patients with a contraindication to OAC or PFO-closure were separately randomized to alternative noncontraindicated treatments (1:1 randomization each). Patients needed to have either large interarterial shunt volume or associated arterial septum aneurysm to enter the trial. The primary efficacy endpoint was recurrence of stroke. Overall, 663 patients were randomized and followed for a mean duration of 5.3 years. No stroke occurred with PFO-closure (238 patients) compared to 14 strokes in the antiplatelet group (14/235 patients) yielding a $\mathrm{HR}$ of $0.03(95 \% \mathrm{Cl}, 0$ to $0.26 ; P<0.001)$; however, just one of the recurrent strokes was deemed to be disabling. The cumulative Kaplan-Meier 5-year estimate of the probability of stroke was $4.9 \%$ in the antiplatelet group. Three recurrent strokes occurred in the anticoagulation group (3/187) corresponding to a cumulative Kaplan-Meier 5-year estimate of the probability of stroke of $1.5 \%$. No formal statistical comparisons were made to compare anticoagulation therapy to antiplatelet treatment or PFO-closure. 
Regarding safety, major procedural device-related complications occurred in 14 (5.9\%) exactly matching the recurrent stroke raw-count in the medical arm over 5 years. In addition, $4.6 \%$ in the PFO-closure group developed atrial fibrillation or flutter compared to only $0.9 \%$ in the antiplatelet arm $(P=0.02)$.

\section{DEFENSE PFO trial}

Device Closure Versus Medical Therapy for Cryptogenic Stroke Patients With High-Risk Patent Foramen Ovale (DEFENSE-PFO) was an investigator-initiated, multicenter, randomized, open-label, superiority trial that compared combined transcatheter PFO closure and medical therapy alone in patients with cryptogenic stroke and high-risk PFO (defined as a PFO with an ASA, atrial septal hypermobility or size $\geq 2 \mathrm{~mm}$ on transesophageal echocardiography). ${ }^{19}$ The trial was conducted at two sites in South Korea from June 2011 through October 2017. PFO closure was performed by experienced interventional cardiologists using the Amplatzer PFO Occluder. All patients received either antiplatelet therapy (single or dual antiplatelets) or anticoagulation (warfarin) therapy chosen by the local investigator. A total of $60 \mathrm{pa}-$ tients were randomized to PFO closure and 60 to medical treatment. Among the 60 patients in the combined PFO closure group, seven declined the intervention. The primary endpoint (composite of stroke, vascular death, or thrombolysis in myocardial infarction [TIMI]-defined major bleeding) during 2 years of follow-up occurred exclusively in the medication-only group (six of 60 patients; 2-year event rate: $12.9 \%$ vs. $0 \%$ [log-rank $P=0.013$ ]; 2-year rate of ischemic stroke: $10.5 \%$ vs. $0 \%$ [log-rank $P=0.023]$ ). Nonfatal procedural complications included development of atrial fibrillation $(n=2)$, pericardial effusion $(n=1)$, and pseudoaneurysm $(n=1)$. There was no case of pulmonary or systemic embolism in any of the two groups.

\section{Meta-analyses}

Following the publications of the new trials, numerous individual-patient data or (network) meta-analyses have been published evaluating the optimal secondary prevention in patients with ischemic stroke and PFO. ${ }^{20-24}$ In brief, these studies indicate that in patients aged $<60$ years, PFO closure probably confers a reduction in ischemic stroke recurrence compared with antiplatelet therapy alone, while it also incurs a risk of persistent atrial fibrillation and device-related adverse events. They also highlight with lower level of evidence that OAC may reduce stroke recurrence in this subgroup of patients compared to antiplatelet therapy. ${ }^{24}$ The latter is mainly based on a post hoc subgroup analysis of the Rivaroxaban Versus Aspirin in Secondary Prevention of Stroke and Prevention of Systemic Embolism in Patients With Recent Embolic Stroke of Undeter- mined Source (NAVIGATE ESUS) trial suggesting a benefit of 15 mg rivaroxaban over aspirin. ${ }^{24}$ However, the Randomized, double-blind, Evaluation in secondary Stroke Prevention comparing the EfficaCy and safety of the oral Thrombin inhibitor dabigatran etexilate vs. acetylsalicylic acid in patients with Embolic Stroke of Undetermined Source (RE-SPECT ESUS) trial failed to confirm such an effect (presented at the World Stroke Congress 2018 in Montreal) and the overall annual risk for bleeding complications was higher in most trials on anticoagulation even in comparable (low-risk) populations than the approximately $1 \% /$ year stroke risk observed in all PFO-trials. This questions whether long-term anticoagulation is a feasible treatment option. Recently, the British Medical Journal Recommendations panel made the following statements regarding PFO patients with cryptogenic ischemic stroke following an extensive diagnostic work-up aged $<60$ years: ${ }^{25}$ (1) for patients who are open to all options, there is a weak recommendation for PFO closure plus antiplatelet therapy rather than anticoagulant therapy; (2) for patients in whom anticoagulation is contraindicated or declined, there is a strong recommendation for PFO closure plus antiplatelet therapy versus antiplatelet therapy alone; (3) for patients in whom closure is contraindicated or declined, there is a weak recommendation for anticoagulant therapy rather than antiplatelet therapy.

Finally, a recent European position paper from multiple scientific organizations (cardiologists, neurologists, stroke physicians) provides the first largely shared, interdisciplinary approach for a rational PFO management based on the best available evidence. ${ }^{26}$ This multidisciplinary position paper indicates a strong recommendation to perform percutaneous closure of a PFO in carefully selected patients aged from 18 to 65 years with a confirmed cryptogenic stroke, TIA, or systemic embolism and an estimated high probability of a causal role of the PFO as assessed by clinical, anatomical, and imaging features. The choice of device should take into consideration that most available evidence has been obtained with the Amplatzer PFO Occluder and GORE HELEX Septal Occluder (not available anymore) or the GORE Cardioform Septal Occluder.

\section{Critical appraisal}

First of all, the authors believe that PFO-closure is a valuable tool in the secondary stroke prevention in selected patients. This was also expressed almost unanimously by the panelists of the FDA-advisory board and was the basis for many positive votes of neurologists in this board. However, in a condition so common (and in most cases completely harmless) in the general population as well in stroke cohorts, there is always fear of 
unwarranted overuse of such an interventional, invasive, and not harmless technique. This is illustrated by anecdotes such as that Bernhard Meier, protagonist of the PC-trial screening his family (primary prevention setting) and subsequently performed two PFO-closures. ${ }^{27}$ It is however also illustrated by the sheer number of procedures performed all over the world even before publication of the three most recent trials. These facts lead to an alerted-in some aspects maybe even to a negativeconception of attentive stroke neurologists.

One concern is the processing and presentation of new trial data. A major source of irritation, at least for the authors of this review, are the multitude of "meta-analyses" and "pooledanalyses" that appear in present days whenever new data become available. ${ }^{28-31}$ Pooling data of trials in different population, using different trial designs and interventions are only of minor help compared to the primary trial data alone. With this in mind, there are more mate-analyses on PFO-trials in the databases than recurrent stroke events in the original trials. In addition, it is interesting to note that even though these analyses include mostly the same trials, many of the publications come up with slightly different results, HR and numbers needed to treat. Based on these analyses different treatment guidelines are presented that often are hard to relate to the initial data (e.g., an upper age limit of 65 years when all randomized trials used an upper age limit of 60 years for inclusion). ${ }^{26}$

The most problematic part of assessing the trial results are so far missing patient-oriented outcome evaluations. It is unclear to the authors why in most cases benefits are not weighted against risks. In light of the overall very low risk for recurrent stroke in all trials (around 1\%/year) even under medical therapy there is not much margin for complications. In the REDUCE-long-term results the rate of pulmonary embolism was increased almost to the extent of the reduction of recurrent stroke. ${ }^{15}$ In CLOSE the raw-count of major device- and/or procedure-related complications exactly equaled the number of recurrent strokes. For the authors it is unclear why these complications are weighted less than efficacy endpoints especially in light of the fact that the majority of outcome events were non-disabling strokes. ${ }^{17}$ No difference was observed with regard of disabling strokes ( 0 event vs. 1 event). A combined endpoint of efficacy and safety endpoints would not have been positive thus emphasizing the need for patient-oriented outcomes. Also, real life registries have repeatedly shown significant procedure-related complication rates that match those of the controlled trials. In a recent publication of a retrospective cohort study using data from New York, California, and Florida total adverse outcomes were observed at the rate of $7 \%$, with new onset of atrial fibrillation/flutter being the most common complication. ${ }^{32}$ In that regard the long-term significance of post- or peri-procedural atrial fibrillation or flutter is largely unknown. Future long-term observations will need to examine whether there is a clinical relevance to this finding, whether there is a higher risk for recurrent episodes, and the impact on overall long-term stroke risk or chronic cardiac changes.

\section{Conclusion and outlook}

All trials demonstrate a very low overall risk of recurrence in young cohorts with cryptogenic stroke and a thorough clinical evaluation. This is of major importance to inform patients and relatives and allows for open and well-considered advice for treatment in this patient cohort. In these patients, recent trials suggest that the already low risk can be lowered further by closure of the PFO in addition to long-term antithrombotic treatment. However, this does not come without some risk emphasizing proper evaluation of appropriate candidates. Several major questions remain for future investigations with age being on important issue. All present trials except for DEFENSEPFO have used an age limit of 60 years and median age in the trials was even lower. However, this limit is arbitrary and needs to be evaluated in future investigations. It is the role of stroke neurologists as well as authorities to closely monitor the usage of this intervention in clinical practice in order to avoid overusage in situations where benefit is not expected. Furthermore, patient-oriented outcome measures as well as long-term evaluations are needed to better evaluate the impact of observed benefits in efficacy against observed complications in the present trials. Until then this intervention should be used reasonably within the examined indication.

\section{Disclosure}

The authors have no financial conflicts of interest.

\section{References}

1. Hagen PT, Scholz DG, Edwards WD. Incidence and size of patent foramen ovale during the first 10 decades of life: an autopsy study of 965 normal hearts. Mayo Clin Proc 1984; 59:17-20.

2. Thompson T, Evans W. Paradoxical embolism. OJM 1930;os23:135-150.

3. Di Tullio M, Sacco RL, Gopal A, Mohr JP, Homma S. Patent foramen ovale as a risk factor for cryptogenic stroke. Ann Intern Med 1992;117:461-465.

4. Lechat $P$, Mas JL, Lascault $G$, Loron $P$, Theard M, Klimczac M, 
et al. Prevalence of patent foramen ovale in patients with stroke. N Engl J Med 1988;318:1148-1152.

5. Webster MW, Chancellor AM, Smith HJ, Swift DL, Sharpe DN, Bass NM, et al. Patent foramen ovale in young stroke patients. Lancet 1988;2:11-12.

6. Mazzucco S, Li L, Binney L, Rothwell PM; Oxford Vascular Study Phenotyped Cohort. Prevalence of patent foramen ovale in cryptogenic transient ischaemic attack and non-disabling stroke at older ages: a population-based study, systematic review, and meta-analysis. Lancet Neurol 2018; 17:609-617.

7. Mas JL, Arquizan C, Lamy C, Zuber M, Cabanes L, Derumeaux $G$, et al. Recurrent cerebrovascular events associated with patent foramen ovale, atrial septal aneurysm, or both. N Engl J Med 2001;345:1740-1746.

8. Overell JR, Bone I, Lees KR. Interatrial septal abnormalities and stroke: a meta-analysis of case-control studies. Neurology 2000;55:1172-1179.

9. Agarwal S, Bajaj NS, Kumbhani DJ, Tuzcu EM, Kapadia SR. Meta-analysis of transcatheter closure versus medical therapy for patent foramen ovale in prevention of recurrent neurological events after presumed paradoxical embolism. JACC Cardiovasc Interv 2012;5:777-789.

10. Almekhlafi MA, Wilton SB, Rabi DM, Ghali WA, Lorenzetti DL, Hill MD. Recurrent cerebral ischemia in medically treated patent foramen ovale: a meta-analysis. Neurology 2009; 73:89-97.

11. Katsanos AH, Spence JD, Bogiatzi C, Parissis J, Giannopoulos $S$, Frogoudaki $A$, et al. Recurrent stroke and patent foramen ovale: a systematic review and meta-analysis. Stroke 2014; 45:3352-3359.

12. Furlan $A J$, Reisman M, Massaro J, Mauri L, Adams $H$, Albers $\mathrm{GW}$, et al. Closure or medical therapy for cryptogenic stroke with patent foramen ovale. N Engl J Med 2012;366:991-999.

13. Meier B, Kalesan B, Mattle HP, Khattab AA, Hildick-Smith D, Dudek $D$, et al. Percutaneous closure of patent foramen ovale in cryptogenic embolism. N Engl J Med 2013;368:1083-1091.

14. Carroll JD, Saver JL, Thaler DE, Smalling RW, Berry S, MacDonald $L A$, et al. Closure of patent foramen ovale versus medical therapy after cryptogenic stroke. N Engl J Med 2013;368:1092-1100.

15. Saver JL, Carroll JD, Thaler DE, Smalling RW, MacDonald LA, Marks DS, et al. Long-term outcomes of patent foramen ovale closure or medical therapy after stroke. N Engl J Med 2017;377:1022-1032.

16. Kent DM, Dahabreh IJ, Ruthazer R, Furlan AJ, Reisman M, Carroll JD, et al. Device closure of patent foramen ovale after stroke: pooled analysis of completed randomized trials. J Am Coll Cardiol 2016;67:907-917.
17. Mas JL, Derumeaux G, Guillon B, Massardier E, Hosseini $H_{\text {, }}$ Mechtouff $L$, et al. Patent foramen ovale closure or anticoagulation vs. antiplatelets after stroke. N Engl J Med 2017;377:10111021.

18. Søndergaard L, Kasner SE, Rhodes JF, Andersen G, Iversen HK, Nielsen-Kudsk JE, et al. Patent foramen ovale closure or antiplatelet therapy for cryptogenic stroke. N Engl J Med 2017; 377:1033-1042.

19. Lee PH, Song JK, Kim JS, Heo R, Lee S, Kim DH, et al. Cryptogenic stroke and high-risk patent foramen ovale: the DEFENSE-PFO trial. J Am Coll Cardiol 2018;71:2335-2342.

20. Stortecky S, da Costa BR, Mattle HP, Carroll J, Hornung M, Sievert $H$, et al. Percutaneous closure of patent foramen ovale in patients with cryptogenic embolism: a network meta-analysis. Eur Heart J 2015;36:120-128.

21. Mir H, Siemieniuk RAC, Ge LC, Foroutan F, Fralick M, Syed T, et al. Patent foramen ovale closure, antiplatelet therapy or anticoagulation in patients with patent foramen ovale and cryptogenic stroke: a systematic review and network metaanalysis incorporating complementary external evidence. BMJ Open 2018;8:e023761.

22. Turc $G$, Calvet $D$, Guérin $P$, Sroussi $M$, Chatellier $G$, Mas $J$, et al. Closure, anticoagulation, or antiplatelet therapy for cryptogenic stroke with patent foramen ovale: systematic review of randomized trials, sequential meta-analysis, and new insights from the CLOSE study. J Am Heart Assoc 2018;7: e008356.

23. Diener HC, Gerloff C, Thaler DE, Wöhrle J. Closure of patent foramen ovale and cryptogenic stroke: unresolved issues. Curr Neurol Neurosci Rep 2018;18:92.

24. Kasner SE, Swaminathan B, Lavados P, Sharma M, Muir K, Veltkamp $R$, et al. Rivaroxaban or aspirin for patent foramen ovale and embolic stroke of undetermined source: a prespecified subgroup analysis from the NAVIGATE ESUS trial. Lancet Neurol 2018;17:1053-1060.

25. Kuijpers T, Spencer FA, Siemieniuk RAC, Vandvik PO, Otto CM, Lytvyn $L$, et al. Patent foramen ovale closure, antiplatelet therapy or anticoagulation therapy alone for management of cryptogenic stroke? A clinical practice guideline. BMJ 2018; 362:k2515.

26. Pristipino C, Sievert H, D'Ascenzo F, Louis Mas J, Meier B, Scacciatella $\mathrm{P}$, et al. European position paper on the management of patients with patent foramen ovale. General approach and left circulation thromboembolism. Eur Heart J 2018 Oct 25 [Epub]. https://doi.org/10.1093/eurheartj/ehy649.

27. Straumann F. Dieses Schirmchen rettet Leben. Berner Zeitung. https://www.tagesanzeiger.ch/wissen/medizin-und-psychologie/Dieses-Schirmchen-rettet-Leben/story/20561568. 2014. Accessed December 18, 2018. 
28. De Rosa S, Sievert H, Sabatino J, Polimeni A, Sorrentino S, Indolfi $\mathrm{C}$. Percutaneous closure versus medical treatment in stroke patients with patent foramen ovale: a systematic review and meta-analysis. Ann Intern Med 2018;168:343-350.

29. Mojadidi MK, Elgendy AY, Elgendy IY, Mahmoud AN, Elbadawi $A$, Eshtehardi $P$, et al. Transcatheter patent foramen ovale closure after cryptogenic stroke: an updated metaanalysis of randomized trials. JACC Cardiovasc Interv 2017; 10:2228-2230.

30. Shah R, Nayyar M, Jovin IS, Rashid A, Bondy BR, Fan TM, et al.
Device closure versus medical therapy alone for patent foramen ovale in patients with cryptogenic stroke: a systematic review and meta-analysis. Ann Intern Med 2018;168:335-342.

31. Saver JL, Mattle HP, Thaler D. Patent foramen ovale closure versus medical therapy for cryptogenic ischemic stroke: a topical review. Stroke 2018;49:1541-1548.

32. Merkler AE, Gialdini G, Yaghi S, Okin PM, ladecola C, Navi BB, et al. Safety outcomes after percutaneous transcatheter closure of patent foramen ovale. Stroke 2017;48:3073-3077. 